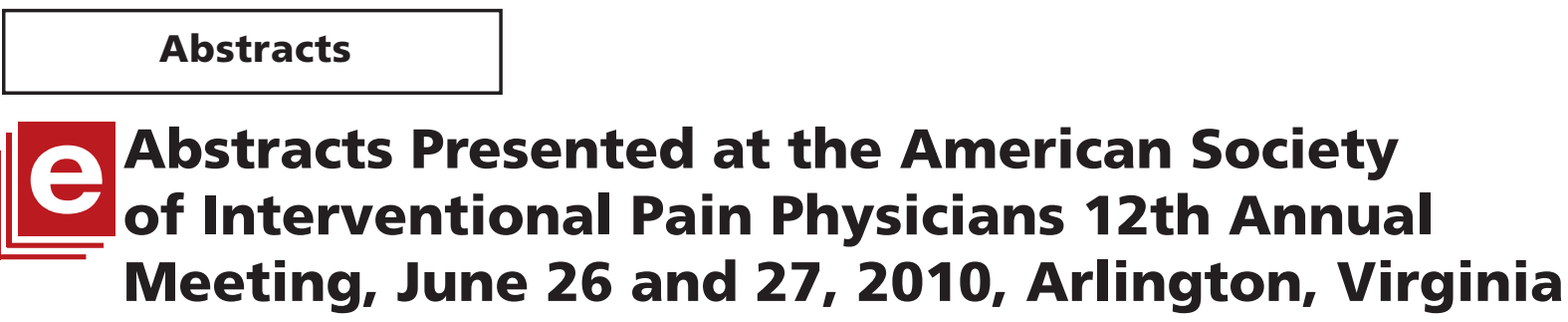

\title{
1. Phantom Radiculopathy Effectively Treated by Fluoroscopically Guided Transforaminal Epidural Steroid Injections
}

Gerard DeGregoris, MD \& Sudhir Diwan, MD

New York Presbyterian Hospital; and Weill Cornell Medical College , New York, NY

Introduction: Lower back and extremity pain in the amputee patient can be challenging to classify and treat (1). Both patients and physicians classically find it difficult to discern between phantom sensation, phantom limb pain, and stump pain, and radicular compression is often not considered (2).

Case Report: We present a case of phantom radiculitis in a 67 -year-old gentleman with new onset $10 / 10$ pain in a phantom extremity superimposed upon a 40-year history of previously stable phantom limb pain. MRI showed a central disc herniation at L4/5 with compression of the traversing left L4 nerve root (Fig. 1). Two fluoroscopically guided left transforaminal epidural steroid injections (one at the level of the $L 4$ and a second at the levels of the L4 and L5 spinal nerve roots) totally alleviated his new onset pain (Fig. 2). At one year post injection, his phantom radiculitis pain was completely gone, though his underlying phantom limb pain remained.

Discussion: Lumbar radiculitis in lower extremity amputee patients may be difficult to differentiate from baseline phantom limb pain. When conservative techniques fail, fluoroscopically guided spinal nerve injection may be valuable in determining the etiology of lower extremity pain. Our experience supports the notion that epidural steroid injections can effectively treat lumbar radiculopathy in lower extremity amputees

\section{References:}

1. Mofidi A, Kotecha A, Banerjee R, Rao CM, Trivedi JM. Sciatica presenting as post-amputation neuroma: Presentation and clinical findings. J Orthop Surg Traumatol 2010; 20:43.

2. Smuck M, Christensen S, Lee SS, Sagher O. An unusual cause of s1 radicular pain. Arch Phys Med Rehabil. 2008; 89:146-149.

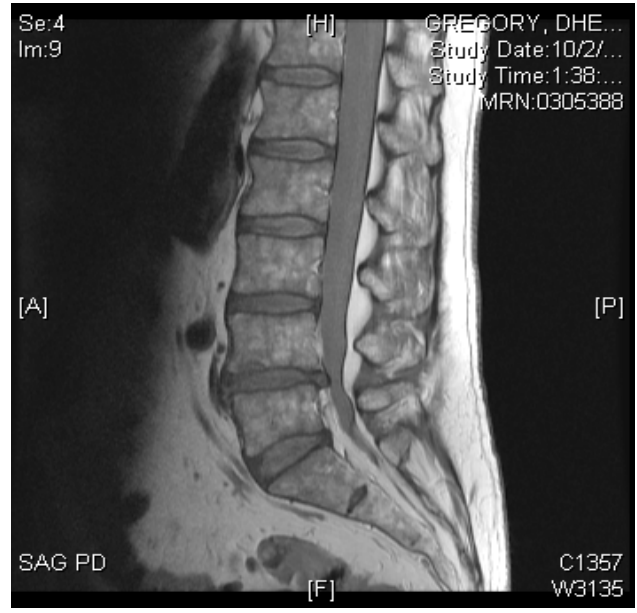

Fig. 1 Saggital MRI showing disc herniation at L4/5 with compression of the traversing left L4 nerve root.

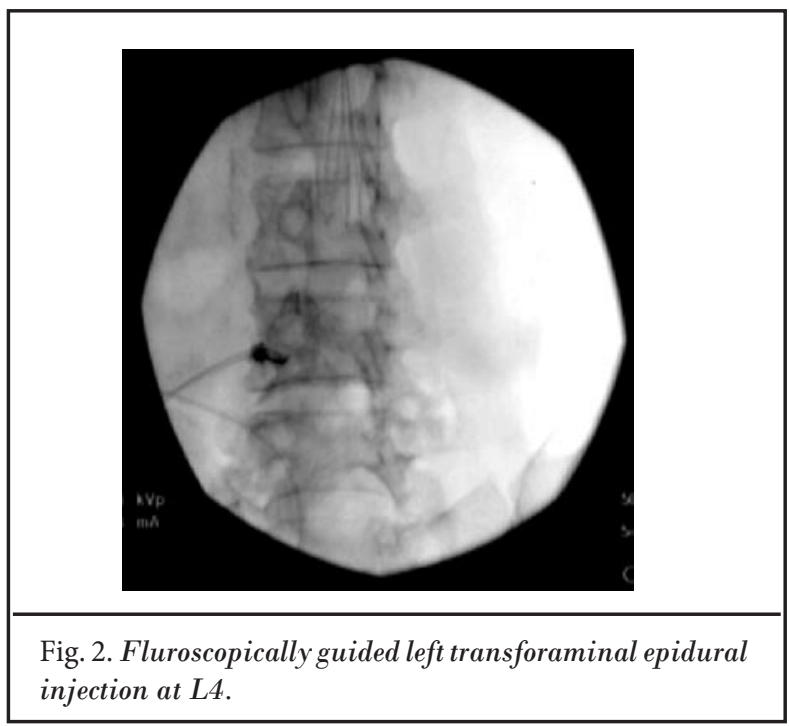




\title{
2. Pulsed Radiofrequency Lesioning of the Right Ilioinguinal Nerve under Ultrasound Guidance in a Pediatric Patient
}

\author{
Ali Emamhosseini, K. Gattu \\ University of Maryland Medical Center, Department of Anesthesiology, Baltimore, MD
}

Introduction: An 11-year-old presented with a 4-month history of intractable right lower-quadrant abdominal pain subsequent to an appendectomy who has failed medical management and has had transient pain relief from past ilioinguinal, iliohypogastric, and epidural catheter placements.

Objectives: To perform and assess the efficacy of an ultrasound-guided pulsed radiofrequency lesioning of the right ilioinguinal nerve in a pediatric patient.

Methods: The ultrasound probe was placed medial to the anterior superior iliac spine (ASIS) and directed toward the umbilicus. The ASIS as well as the muscle layers including the external oblique (EO), internal oblique (IO) and transversus abdominis (TA) were identified. The right ilioinguinal nerve was located between the $1 \mathrm{O}$ and the TA, and roughly $8 \mathrm{~mm}$ medial to the ASIS. Under ultrasound guidance we advanced a 5 $\mathrm{cm}, 2$-gauge angulated tip radiofrequency cannula until the needle was overlying the nerve. The radiofrequency probe was then inserted into the cannula and the needle tip position was reconfirmed. Two rounds of pulsed radiofrequency lesioning were done at 42 degrees Celsius for 120 seconds. Then a therapeutic solution of $3 \mathrm{~mL}$ of Bupivacaine $0.25 \%$ and $1 \mathrm{~mL}$ of Kenalog $40 \mathrm{mg} / \mathrm{mL}$ was given demonstrating excellent spread under ultrasound imaging.

Results: The patient is roughly 2 months post procedure, pain free per phone follow-up and has not returned to the clinic or required further treatments.

Conclusions: Pulsed radiofrequency in the pediatric population maybe an efficacious treatment option in cases of ilioinguinal neuralgia refractory to conservative management. 


\title{
3. Test Results Showing Bench Reliability on Catheter Dislodgement for Ascenda ${ }^{\mathrm{TM}}$, Medtronic Neuromodulation's Next-Generation Catheter
}

\author{
Karl Hokanson, BSME, MSME, Michael Metzler, BSME, Julie Kopp, BS, Andrew Servi, BS, Amy \\ Gjoraas, BS
}

Medtronic Neuromodulation, Minneapolis, MN

Background/Objectives: The SynchroMed®II infusion system is a programmable, implanted medical device designed to administer prescribed drugs to the intrathecal space over an extended period of time. Components include the implantable pump and catheter, a programmer, and accessories. AscendaTM, a next-generation catheter for the infusion system, was recently developed, offering a robust, multi-layer, polymer braided design; increased anchoring strength; and an enhanced catheter-to-catheter connection. The objective of the present study was to evaluate the Ascenda catheter with respect to several known failure modes for dislodgement (the migration of the catheter from the intrathecal space, resulting in drug delivery interruption). This can occur even when an anchor is sutured to the site where the catheter exits the fascia. The catheter can migrate relative to the fascia due to:

- a "sweeping motion" between skin and fascia, pulling the catheter out in front of the anchor, or

- tensile forces from body motion proximal to the anchor, pulling the catheter through the anchor.

Description: To evaluate catheter dislodgement due to a "sweeping motion" between the skin and fascia, an anchored Ascenda catheter was exposed to oscillatory force of $0.70 \mathrm{lbf}$, perpendicular to the catheter axis, based on a published study that investigated the force required to remove a lumbar extradural catheter from a series of patients. Dislodgement, when it occurs distal to the anchor, is expected to happen within one year after implant. The anchored catheter was exposed to this force for a minimum of 120,450 cycles, based on a study that evaluated activities of daily living (eg, sitting, standing) and the number of cycles these activities could impart in one year.

To evaluate catheter migration and cyclic axial fatigue performance due to tensile stresses from body motion proximal to the anchor, the Ascenda catheter anchor was exposed to a cyclic axial fatigue test for a minimum of 850,000 cycles at a displacement of 0.27 inches.

During attempted dislodgement due to a "sweeping" motion, the Ascenda catheter withstood 250,000 cycles (more than twice the requirement) without displacement of the anchor. During tensile cyclic fatigue exposure, the Ascenda catheter withstood 1,700,000 cycles (twice the requirement) without leak, occlusion, or movement of the catheter relative to the anchor.

Significance: These bench test results, observed in a simulated in vivo environment, illustrate the robustness of the Ascenda catheter with respect to dislodgement, above and beyond established design requirements. The catheter demonstrated marked resistance to breakage and occlusion during similarly stringent bench testing of those potential failure modes. When considered collectively, these test results provide evidence of a highly reliable and robust catheter design. 


\title{
4. Minimally Invasive Spine Surgery: Interventional Pain Physician's Perspective
}

\author{
Hey-ran Choi, Pyung-Bok Lee, Kyung-Hoon Kim, MD, PhD* \\ Department of Anesthesia and Pain Medicine, School of Medicine, Seoul National University, Seoul, Korea \\ Department of Anesthesia and Pain Medicine, School of Medicine, Pusan National University, Busan, Korea*
}

The term "minimally invasive surgeries and techniques" has been used in various medical fields including interventional pain management. It is essential for the patients with spine pain to have the correct diagnosis and initial treatment by a pain physician before conventional surgery. Over $95 \%$ of the degenerative spinal disorders can be managed without surgery, and the majority of those that require surgical intervention can benefit from minimally invasive spine surgery (MISS).

Minimally invasive percutaneous endoscopic discectomy is a cutting-edge technique of MISS for the surgical treatment of discogenic spine pain. The mechanisms of pain associated with the herniated nucleus pulposus are mechanical compression and/or chemical irritation of the dorsal root ganglia which results in changes in the peripheral as well as central nervous system. Thus the 3 main mechanisms of pain reduction are nerve root decompression for herniated portion of nucleus pulposus, reduction of chemical irritants by continuous irrigation, and denervation for ingrowing nerve fibers into the inner part of annulus fibrosus.

One thousand twenty-four patients who failed epidural steroid injections for persistent neck/back and arm/leg pain underwent fluoroscopically guided percutaneous endoscopic cervical and lumbar endoscopic discectomies over the last 10 years in the Pain Clinic, Pusan National University Hospital. All operations were performed under local anesthesia with a lidocaine patch application on the predicted insertion site of the needle and working channel and intravenous analgesia using 100 $\mathrm{gg}$ of fentanyl and $30 \mathrm{mg}$ of ketolorac. The direction and distance from the midline of a 17-gauge needle was determined by the direction of the herniation. After confirmatory discography with a mixture of $4 \mathrm{~mL}$ of the contrast medium and $1 \mathrm{~mL}$ of $\mathrm{In}$ - digo carmine, serial dilation was performed for the insertion of the working channel. Before the anulotome, 3 or $4 \mathrm{~mL}$ of intraannular injection of $1 \%$ lidocaine was done inside the working channel. The extracted volume was determined by pre-operative magnetic resonance image and was sufficiently confirmed by the patient's coughing at the end of the operation through the direct endoscopic field.

The mean age was $55.7 \pm 15.5$ (range from 17 to 89 ) years and the mean hospital stay was $1.9 \pm 1.5$ days. The mean volume of extracted nucleus pulposus was $1.8 \pm 1.2 \mathrm{~mL}$. Patients could felt comfortable just after decompression during the operation. The positive straight leg raise test was turned into a negative immediately after the operation. They could ambulate after checking the computed tomography immediately after the operation. Some of the complications observed were 189 cases of piriformis syndrome, 5 cases of incomplete decompression, 3 cases of burning sensation on the femoral nerve distribution (sunburn phenomenon), 2 cases of re-herniation, 2 cases of foot drop, and one case of hematoma around psoas muscle. The follow-up schedule was one, 4, 8, and 12 weeks after the operation if there is was any complication. During the followup period, an anticonvulsant was prescribed for the dysesthesia on the extremity.

Summary: MISS, especially, percutaneous endoscopic discectomy should be offered for patients with discogenic spine pain before conventional spine surgery is considered. Finally, interventional pain physicians should learn and master this technique.

Key words: Discectomy, endoscope, minimally invasive surgical procedures, spine. 


\title{
5. Intraforaminal Location of the Great Radiculomedullary Artery (Artery of Adamkiewicz): A Cadaver Study
}

\author{
Andrzej C. Kroszczynski, MD1, Kevin Kohan, DO'1, Marek Kurowski, MD², \\ Sherry A. Downie, PhD ${ }^{1,3}$, Todd R. Olson ${ }^{3}$, Mark A. Thomas ${ }^{1}$ \\ 1Albert Einstein College of Medicine, Department of Physical Medicine and Rehabilitation, Bronx, NY \\ 2 Penn State Hershey, Department of Anesthesiology, Pain Medicine Division, Hershey, PA \\ 3Albert Einstein College of Medicine, Department of Anatomy and Structural Biology, Bronx, NY
}

Background: Damage or injection of steroids to the Artery of Adamkiewicz (AKA) or anterior radiculomedullary artery (ARMA) has been postulated as the mechanism of paraplegia following transforaminal epidural steroid injections (TFE$\mathrm{SI})$. In order to avoid catastrophic complications during TFE$\mathrm{SI}$, it is vitally important to know the intraforaminal anatomy of AKA. In a limited cadaveric study Alleyne showed its location in the superior or midportion of the foramen and anterosuperior to the dorsal root ganglion-ventral root (DRG-VR) complex. In a radiological study of 113 ARMA arteries, Murthy found the AKA to be located in the upper third of the foramen in $88 \%$ of cases.

Objectives: To examine and precisely measure the intraforaminal location of AKA and its relationship to the DRG-VR complex in a large cadaveric dissection study.

Methods: AKAs and thoracolumbar ARMA arteries were visualized in 23 embalmed cadavers. Access to the anterior surface of the spinal cord was made via anterior corpectomy with sparing of the posterolateral vertebral bodies in order to preserve the intervertebral foramina. Each AKA and ARMA was followed to its intervertebral foramen. The foramen was subsequently opened in the coronal plane. The intraforaminal locaction of the artery, its diameter, and its relation to the foraminal structures were examined and measured.

Results: 23 GRAs and 15 ARMAs were identified in the thoracolumbar intervertebral foramina (T4 - L2). Of these, 29 (74\%) arteries were located in the upper $1 / 3$ of the foramen, $9(23 \%)$ were located in the middle, and $1(3 \%)$ artery was located in the lower 1/3. In relation to the DRG-VR, 21 (54\%) arteries were located anterosuperiorly, 16 (41\%) anteriorly, and 2 (5\%) anteroinferorly to the DRG-VR complex. The average distance from the superior border of the artery to the upper pedicle was 1.2 $\mathrm{mm}(0-4.46 \mathrm{~mm})$. The average distance from the inferior border of the artery to the lower pedicle was $5.09 \mathrm{~mm}(2.15$ -

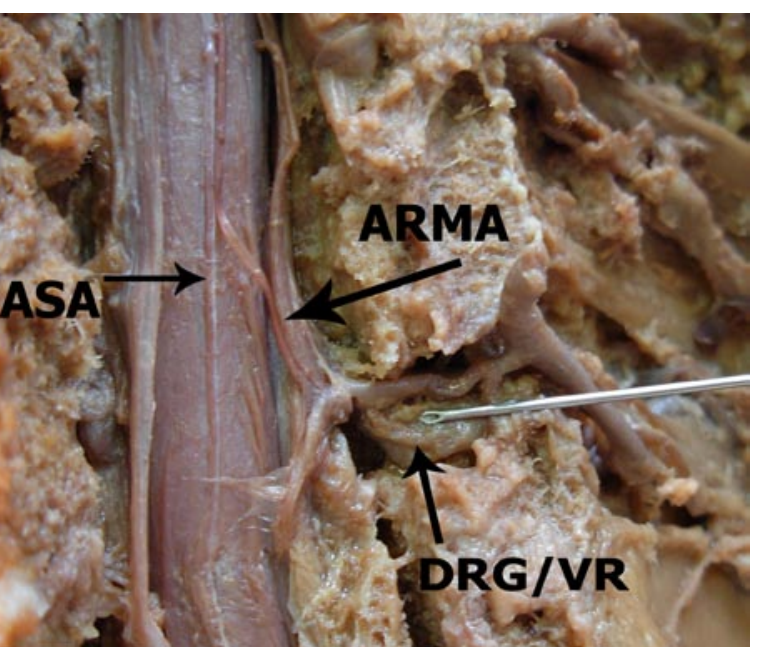

Fig. 1. Anatomy of the neuroforamen with a 22-g needle added for comparison. DRG/VR: dorsal root ganglion/ventral root complex, ASA: anterior spinal artery, ARMA: anterior radiculomedullary artery.

$10.45 \mathrm{~mm})$. The average intraforaminal artery diameter was $1.32 \mathrm{~mm}(0.96-1.91 \mathrm{~mm})$ for AKA and $1.04 \mathrm{~mm}(0.84-1.36$ $\mathrm{mm})$ for ARMA.

Discussion/Conclusions: AKAs and ARMAs were showed to be predominantly located in the upper $1 / 3$ of the foramen, anterosuperior or anterior to the DRG-VR complex. Based on a better understanding of the intraforaminal position of the AKA and ARMA, it is proposed that an infraforaminal injection may be adopted as a safe alternative to the current subpedicular TFESI technique. 


\title{
6. Left S2 Adamkiewicz Artery: A Case Study
}

\author{
Andrzej C. Kroszczynski, MD1, Kevin Kohan, DO', Marek Kurowski, MD², \\ Sherry A. Downie, PhD ${ }^{1,3}$, Todd R. Olson ${ }^{3}$, Mark A. Thomas ${ }^{1}$ \\ Affiliations: \\ 1Albert Einstein College of Medicine, Department of Physical Medicine and Rehabilitation, Bronx, NY \\ 2 Penn State Hershey, Department of Anesthesiology, Pain Medicine Division, Hershey, PA \\ 3Albert Einstein College of Medicine, Department of Anatomy and Structural Biology, Bronx, NY
}

Background: The first published case series of paraperesis as a complication of transforaminal epidural steroid injections by Houten and Errico included a case that developed following left $\mathrm{S} 1$ injection. The postulated pathomechanism was the injection of steroids or damage to the artery of Adamkiewicz (AKA) or anterior radiculomedullary artery (ARMA) and subsequent spinal cord infarction. To our knowledge, the AKA has not been described at the S1 level and below. Desproges-Gotteron Arteries supply the L5 and S1 roots and the spinal cord, however, not via the anterior spinal artery.

Objectives: To describe an AKA entering via the left S2 foramen.

Methods: The embalmed female African-American cadaver was placed supine. After removing the internal organs, anterior corpectomy was performed from T2 to S1. The posterior longitudinal ligament was removed and the dura was opened. The Anterior Spinal Artery (ASA) was carefully examined from $\mathrm{T} 2$ to the conus medullaris. The only arterial branch supplying the ASA anastomosed at the T12 level and originated at the sacral level. Gentle traction was applied to the left S1, S2, S3, and $\mathrm{S} 4$ roots from the pelvic cavity. The movement of the artery was observed only with S2 root traction. The left S2 foramen was opened from the medial side. The artery was tracked down to the S2 dorsal root ganglion/ventral root complex.

Results: The AKA entered the vertebral canal via the left S2 foramen and anastomosed with the anterior spinal artery at the T12 level.

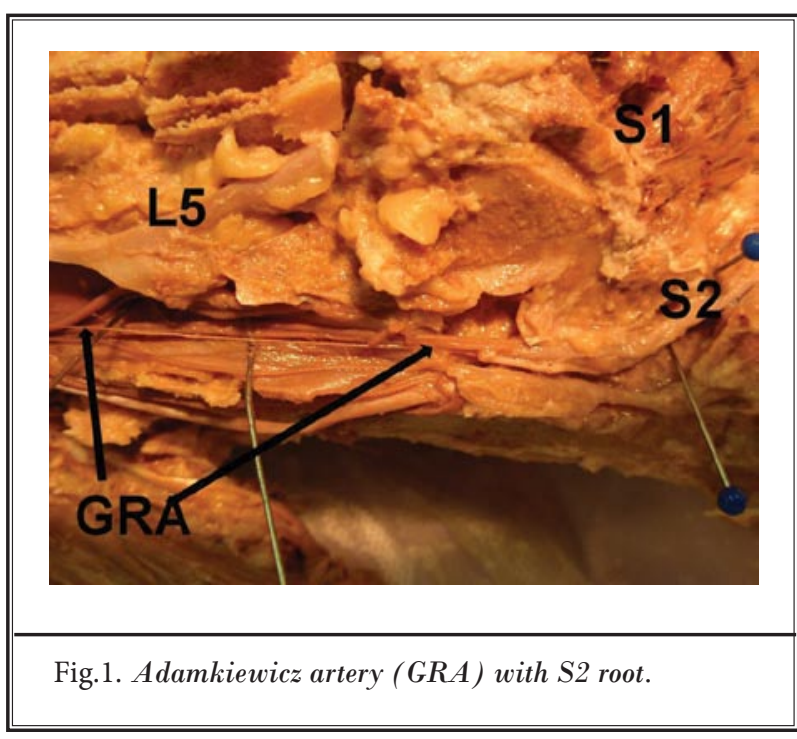

Discussion/Conclusions: We found only one anterior radiculomedullary artery directly anastomosed with the ASA from T2 to $\$ 4$ and it exhibited the characteristic hairpin turn just prior to joining the ASA. Thus, by convention, we called it the AKA. The finding of the AKA in a sacral foramen may explain paraplegia after left $\mathrm{S} 1$ transforaminal steroid injections as reported by Houten. To our knowledge, this is the first report of AKA entering as low as $\mathrm{S} 2$. 


\section{Radiofrequency Neuromodulation of the Iliohypogastric Nerve and Genitofemoral Nerve Block for Recalcitrant Chronic Pelvic Pain}

Cyril N. Philip, MD, Kenneth D. Candido, MD

Department of Anesthesiology and Pain Management, Advocate Illinois Masonic Medical Center, Chicago, IL

A 20-year-old morbidly obese female presented with a 2-year history of burning dysesthesias bilaterally in the groin and vaginal areas. This malady limited the patient's ability to exercise and was exacerbated by urination and cutaneous palpation. She was subjected to multiple pudendal nerve blocks at an outside institution with no resolution of symptoms. Contrarily, our physical examination and history suggested the iliohypogastric nerve to be the source of her distress. Pulsed radiofrequency (PRF) neuromodulation, followed by local anesthetic and dexamethasone injections resolved the pain in the left groin and vaginal areas.
After further diagnostic nerve blocks, the right sided groin pain was attributed to a neuropathy associated with the genitofemoral nerve and was subsequently blocked with local anesthetic and dexamethasone. The patient has experienced pain relief for 5 months and consequently is able to partake more thoroughly in her aquatic therapy. We believe a multimodal approach incorporating pulsed radiofrequency neuromodulation can facilitate the recovery of activities of daily living especially in patients suffering from chronic pelvic pain. 


\title{
8. How to Obtain a Clear Fluoroscopic Lateral View for Cervical Epidural Steroid Injection
}

\author{
YiLi Zhou MD, PhD \\ Comprehensive Pain Management of North Florida, Lake City, FL
}

Object: To investigate a new method that will provide a clearer lateral fluoroscopy view of the Tuohy needle tip in the cervical epidural space.

Background: Cervical epidural steroid injections (CESI) are being performed widely to treat neck pain due to cervical radiculopathy. However, some serious complications, such as direct spinal cord damage, have been reported. To decrease the risks of complications, it is critical to obtain a clear lateral view with C-arm fluoroscopy to confirm the Tuohy needle tip in the epidural space before the medications are injected. However, it has been difficult to obtain a clear lateral view fluoroscopy picture because most of times, the needle tip is buried in the dark shadow of the shoulders. The current study is designed to find a new method to obtain a clearer lateral fluoroscopy view of the Tuohy needle tip in the cervical epidural space.

Methods: This prospective study was approved by IRB in Comprehensive Pain Management of North Florida. Five patients participated in the study after an informed consent was signed by each of the participants. The patient was positioned prone on the fluoroscopy table. A 20-gauge, 3.5-inch Tuohy needle was introduced into the C7-T1 epidural space under the guidance of the fluoroscopy. An AP view was first obtained with beam of X-ray apparel to the $C 7$ and $T 1$ spinous process and the Tuohy needle with the needle at the middle of the fluoroscopy image. The C-arm fluoroscopy was then rotated to a lateral position. In this position, the junction between the shoulder and neck is usually in the middle of the fluoroscopy tubes (half shoulder-half neck position). A first lateral fluoroscopy picture was taken. The needle tip is usually in the low one-third to middle position in the fluoroscopy picture in a caudal cephaloid direction. The fluoroscopy machine was then slightly moved to the leg direction for about an inch resulting in more shoulder and less neck between the fluoroscopy tubes (more-shoulder position). A second lateral fluoroscopy picture was taken. Now the needle tip position should be moved to the upper one-third position in the caudal cephaloid direction in the fluoroscopy picture. After a clear view of the needle tip and spread of the contrast was obtained, $80 \mathrm{~mL}$ of Kenalog with $0.2 \mathrm{~mL}$ of $1 \%$ preservative free Lidocaine was injected into the epidural space for the treatment purpose. Then the fluoroscopy was moved to original "half shoulder-half neck position." A repeated lateral fluoroscopy picture was taken.

Results: In 4 of the 5 subjects with a body weight of more than 130 pounds, all the lateral fluoroscopy pictures obtained in the traditional "half shoulder-half neck position" showed very poor needle tip localization. However, the lateral fluoroscopy pictures obtained from the "more-shoulder" position showed a clearer needle tip position and clear spread of contrast in the epidural space. After the fluoroscopy moved back to the original "half shoulder-half neck position," the contrast was difficult to see in the lateral view. 


\title{
9. Phenol vs. Radiofrequency Lumbar Facet Joint Rhizotomy: Comparison of Clinical Efficacy
}

\author{
Yili Zhou, MD, PhD., Stephen Irwin, MD, MBA, Matthew Barnes, PA-C
}

Comprehensive Pain Management of North Florida, Lake City, FL

Objective: The object of this study was to compare the clinical efficacy of phenol vs. radiofrequency lumbar facet joint rhizotomy.

Background: Radiofrequency facet joint rhizotomy has been used extensively to treat lumbar facet joint syndrome with moderate evidence to support its clinical efficacy. Phenol is a neurolytic agent and has been used to treat various pain conditions. Our previous study found that phenol lumbar facet joint rhizotomy is relatively safe. However, the clinical efficacy of phenol lumbar facet joint rhizotomy has not been well studied. The current study is designed to compare the clinical efficacy of phenol vs. radiofrequency lumbar facet joint rhizotomy.

Methods: This is a retrospective chart review. From January 2009 to January 2010 all patients who came to our practice with possible lumbar facet joint syndrome underwent 2 consecutive diagnostic medial branch blocks (MBB). If the patients had more than $50 \%$ of pain relief after each of the 2 diagnostic MBB without any leg weakness they were assigned for phenol or radiofrequncy rhizotomy. All phenol rhizotomies were performed by physician A and RF rhizotomies were performed by physician $\mathrm{B}$.

For phenol rhizotomy, multiple 25-gauge, 3.5-inch spinal needles were placed at the junction between the superior articular process and transverse process (Scotty Dog's eye) depending on the area involved in pain. $0.5 \mathrm{~mL}$ of Omnipaque was first injected to make sure no contrast spread to the adjacent nerve roots as confirmed by AP and lateral fluoroscopy. $0.5 \mathrm{mLof}$ $5 \%$ aqueous phenol was then injected through each needle to block the target nerves.

For RF rhizotomy, A Cosman G4 radiofrequency generator and 22-G Cosman RF needles were used. The needles were placed in the same way as for the phenol rhizotomy except that the needles were inserted in a more caudal-cephaloid direction so that the needle tip will be more parallel to the target nerves. Each level was tested for sensory as well as multifidus motor stimulation. RF lesion was made by raising the temperature of the electrode tip to $80 \mathrm{C}$ for 90 seconds.

Most of the patients had a follow-up visit about one month after the procedures. During the follow-up visits, the patients were asked for the percentage of pain relief from the rhizotomy and side effects, if any, were also documented.

Results: From January 2009 to January 2010, 62 cases (42 females, 21 males) of phenol rhizotomies were performed. Mean age of the group was 58.8 years old with an average VAS pain score of 5.8 at an 11-point scale. Fifty-five cases (40 females, 15 males) of RF rhizotomies were performed. Mean age of the group was 61.1 years old with an average VAS pain score of 5.5 at an 11-point scale. For the phenol group, 47 out of 61 (75\%) had a follow-up visit in one month. Among them 37 patients $(79 \%)$ reported various degree of pain relief. For the RF group, 34 out of $55(61 \%)$ had follow-up visit in one month. Among them 28 (82\%) reported various degrees of pain relief. The average pain relief was $52 \%$ and $54 \%$ for phenol and RF group respectively $(P>0.05)$. No significant side effects such as skin lesion, muscle necrosis, leg weakness, incontinence, systemic infection, or any other side effects were reported in either group.

Discussion: The results of this study suggest that phenol rhizotomy for lumbar facet joint syndrome is as effective as radiofrequency rhizotomy at a one month follow up. Both methods are safe. However, this study is a retrospective chart review, with only one-month follow-up. The patients were not randomly assigned. There were different doctors performing each technique. A prospective study is warranted to further investigate the clinical efficacy for phenol lumbar facet joint rhizotomy. 


\title{
10. Minimally Invasive Lumbar Decompression Using the MILD Procedure for Debilitating Lumbar Central Canal Stenosis
}

\author{
Sheri L. Albers DO, and Wade Wong DO \\ University of California at San Diego, San Diego, CA
}

Background: In patients over 55 years of age, lumbar spinal stenosis is one of the most common indications for surgical intervention. Depending on the degree of stenosis this process can be devastating to a patient's motor, genito-urinary, and GI functions. Each year in the United States, 1.4 million people are diagnosed with lumbar spinal stenosis. This number is growing secondary to aging "baby boomers." In fact, there are approximately twice as many patients diagnosed with lumbar spinal stenosis as compared to patients diagnosed with vertebral compression fractures.

With the exception of open surgery, therapeutic options have been limited for patients with lumbar central spinal stenosis. However, there are significant problems with open surgery. The length of hospitalization is varied. There is a wide range of post procedural pain, hematoma formation, and scarring. The overall surgical complication rate is approximately $15 \%$ and includes dural tears, infection, and instability. Patients can develop failed back syndrome. Additional surgery may be required. Recovery can be prolonged. The need for General Anesthesia also increases risks and costs for patients.

Purpose: To access the efficacy of the mild Procedure (Vertos Medical Inc., 11 Columbia Suite B, Aliso Viejo, CA 92656): a new fluoroscopically guided, minimally invasive lumbar decompression procedure preformed in conjunction with an epidurogram.

- To evaluate the ability of the MILD procedure to effectively reduce the degree of central canal stenosis by removing ligamentum flavum and small amounts of lamina, using a percutaneously placed canula through which bone and tissue sculpturing microtools are passed.
- To compare the MILD procedure complication rate to the complication rates of open surgical procedures.

- To assess patient outcome and satisfaction following the MILD procedure.

Methods and Materials: Twelve patients varying in age from 57 to 88 with a history of severe disability from neurogenic claudication due to central canal stenosis, already having failed conservative measures, were assessed using VAS pain scales and Oswestry Disability Indices. The patients were assessed at the following intervals: pre-procedure, and one week, 3 weeks, 6 weeks, and 12 weeks post procedure. Each procedure was performed with a local anesthetic, moderate sedation, and under fluoroscopic guidance.

Results: The average pre-procedure VAS pain scale was $7.75 / 10$, dropping to 2.5/10 at 3 weeks and 2/10 at 12 weeks. The average Oswestry Disability Index scale pre-procedure was 48 (severe) dropping to 23 (mild) at 3 weeks and to 17 (mild) at 12 weeks. The latter represented a 36 point difference on a scale where a 15 point difference is considered significant. There was minimal soft tissue trauma. The patients tolerated the procedure well. There were no complications. Recovery time averaged less than 3 hours.

Conclusion: In patients who are disabled from neurogenic claudication due to central canal stenosis, who have failed conservative measures, and who do not want an invasive surgical procedure, the MILD procedure offers a safe, effective, comparable treatment option. The open surgical methods have a historical complication rate of $15 \%$, while in this small patient population, there were no complications. 


\section{Comparative Study of Epidural Steroid Injection versus mild (Minimally Invasive Lumbar Decompression) Procedure in Patients Diagnosed with Symptomatic Moderate to Severe Lumbar Central Canal Stenosis}

Lora Lee Brown, MD

Coastal Orthopedics \& Sports Medicine, Bradenton, FL

Background: Various minimally invasive and surgical options are used to perform lumbar decompressive procedures. The goal of the mild procedure is to effect a decompression with minimal surrounding tissue disruption. The standard medical therapy widely used to treat symptomatic central canal spinal stenosis is a combination of pain medicine, epidural steroids (ESI), and/or physical therapy. Where conservative therapy fails, the common surgical procedures involve various techniques that range from an insertion of an interspinous decompression implant to moderate endoscopic or major open surgery. These all may result in various amounts of damage to large areas of tissue, muscle, ligaments, and bone in the back. Recovery from the major surgical procedures is painful and can require several months of low activity and rest.

Objectives: The purpose of this single-center, randomized, prospective, double-blind clinical study is to assess the clinical application and outcomes with mild devices versus epidural steroid injection in patients with symptomatic moderate to severe central canal spinal stenosis.

Methods: Patients with symptomatic lumbar spinal stenosis who meet the study enrollment criteria are randomized to either ESI or the mild procedure. Patients are treated with the mild procedure under radiographic image guidance as described in the mild product labeling, or with ESI. Neither the enrolling physician nor the subject is aware of the subject's ultimate treatment group. These patients are followed for a period of up to 6 months in order to assess patient outcomes. Complete enrollment will include 40 adult patients at one study center.
Documentation of concomitant and pain medication usage, neurological examination, Visual Analog Score (VAS), Quality of Life (SF-12), Oswestry Disability Index (ODI), and Zurich Claudication Questionnaire (ZCQ) are made during the scheduled screening/baseline physical examination prior to study treatment. Patient follow-up via telephone survey and office visits include documentation of the same above measures and any medication changes. Device-related and significant adverse events are documented throughout the study period.

Results: Enrollment is under way for this study, and is anticipated to be completed during the first half of 2010. The mild and traditional injection therapy groups will be analyzed separately for safety and effectiveness, and the 2 groups will be compared. Outcome measures to be evaluated include:

- $\quad$ Ability to perform treatments as planned free of technical complications

- $\quad$ Acceptable safety, consistent with other similar procedures

- $\quad$ Subject success, supported by procedure completion and effectiveness

- $\quad$ Changes in back pain as measured by a 10-point VAS

- $\quad$ Function as measured subjectively by the ODI, ZCQ, and Work Production Index patient questionnaires

- $\quad$ Qualify of Life Physical Component Score (PCS) as measured by the SF-12 short form survey

- Changes in neurological status as determined through physical examination

- $\quad$ Changes in pain medication requirements

Discussion and Conclusion: The discussion will compare safety and effectiveness of the 2 study groups. The conclusion will summarize and assess the study findings. 


\title{
12. Pulsed Radiofrequency on L2 Dorsal Root Ganglion as a Therapeutic Method for Lumbar Discogenic Pain: One Year Follow-up Study Involving 26 Patients
}

\author{
Carlos Tucci, MD ${ }^{1,3}$, Jamir Sardá Jr., PhD, ${ }^{1,4}$ \\ 1Singular Pain Clinic, Campinas, SP, Brazil \\ 2Integrated Center for the Treatment of Pain, Albert Einstein Hospital, SP, Brazil \\ 3PUC - Catholic University, Campinas, SP, Brazil \\ 4 Vale do Itajaí University, Univali, SC, Brazil
}

Fabricio Dias Assis, MD¹, Charles Amaral de Oliveira, MD', Fabíola Peixoto Minson, MD ${ }^{1,2}$,

Chronic low back pain can be defined as pain that lasts beyond 3 months. The high prevalence of this condition in the general population incurs substantial social and economic costs to society. Discogenic pain is one of the most common causes of chronic low back pain, along with facet arthrosis and sacroiliitis. According to the IASP definition, positive provocative discography is the basis for confirming a diagnosis of discogenic low back pain. Patients often present with low back pain, and MRI shows a black disc with or without a high-intensity zone. L2 dorsal root ganglion diagnostic blocks have emerged as an alternative to discography. This study suggests that the afferent sympathetic fibers emerging in the $\mathrm{L} 2$ dorsal root ganglion are pathways of nociceptive transmission. Pulsed radio frequency (PRF) for discogenic pain is a relatively new technique, compounded by the paucity of evidence available.

Objective: This study aims to confirm the diagnostic effect of blocking the L2 dorsal root ganglion and the efficacy of applying PRF to its root as a treatment option for discogenic low back pain. To evaluate the efficacy of PRF on L2 dorsal root ganglion for chronic discogenic low back pain, this study examined the outcomes of 26 patients who underwent this procedure between 2007 and 2009. The study was conducted in a private pain clinic, located in Mogi Mirim, São Paulo, Brazil.

Methods: This is a cross-sectional retrospective study of a convenience sample of 26 patients based on physical examination, magnetic resonance imaging findings, and targeted $L 2$ dorsal root ganglion diagnostic block. Patients who had had such diagnostic blocks and presented with $50 \%$ pain relief on the visual numeric scale (VNS) underwent PRF on bilateral L2 dorsal root ganglion. Pain was evaluated using the visual numeric scale pri- or to the intervention with follow-up scoring at 1 month, 3 months, 6 months, and 12 months. Data from the Oswestry Disability Questionnaire (ODI) and SF-36 were also collected. Data analyses were conducted using descriptive and inferential statistics, T-test and the Friedman nonparametric test using SPSS-11.

Results: Patient mean age was $47(S D=15.26)$; pre-intervention mean pain intensity, $7.4(S D=2.4)$; mean pain duration, 131 months $(S D=112) ; 65 \%$ of the participants were female. At 1-month follow-up $(\chi=2.1, S D 2.0)$, statistical analysis revealed a significant decrease in pain $(P<0.001)$, which lasted up to 12 months $(\chi=3.3, S D=2.5)$. The Oswestry Disability Index score prior to intervention was $52.05(S D=51.0)$ and at one year post-surgical intervention, $35.72(S D=33)(t=3.10$, $P=0.01)$. No SF-36 scale scores were assessed until a year later, when they were found to be around $50 \%$, ranging from $41 \%$ to $61 \%$.

Conclusion: Pulsed radiofrequency on the L2 dorsal root ganglion is an effective, non-specific therapeutic method for discogenic low back pain. This procedure produced significant results with $54 \%$ pain relief for up to one year. The $31 \%$ reduction in disability most likely stemmed from the decrease in pain. About average quality of life scores (50\%) are lower in comparison with those of healthy subjects (mean scores around $80 \%$ ). The quality of life scores of this population may have been affected by other factors beside pain.

Key words: Low back pain, discogenic pain, L-2 DRG, disability, quality of life 


\title{
13. Safe Needle Entry Angle for Cervical Transforaminal Epidural Steroid Injections
}

\author{
Boqing Chen, MD, PhD¹, Pinella Holder, DO¹, Jeffrey Chacko, MD¹, and Daniel Chen²
}

1Center for Advanced Pain Management and Rehabilitation, LLC, Newark, NJ

2Emory University, Atlanta, GA

Purpose: To investigate a practical and safe needle entry angle for cervical transforaminal epidural steroid injections using the oblique approach with fluoroscopic guidance.

Objectives: Cervical transforaminal epidural steroid injections using an anterior oblique approach under fluoroscopic guidance have been used in the treatment of neck pain with radiculopathy for years. There have however been no studies to our knowledge describing the optimal needle entry angle for these injections. Improper needle placement inside the neuroforamina not only diminishes the procedure's therapeutic and diagnostic value but also increases the likelihood for complications, including vascular penetration and nerve injuries. This study was designed to determine whether there is a safe angle of entry in the oblique view based on the measurement across sectional cervical spine CT scans as well as confirmation during actual cervical transforaminal injections that were performed.

Materials and Methods: Forty-seven consecutive patients under went cervical post discography CT scans from March 2009 to December 2009 in a free standing imaging center. There were 29 females and 18 males with ages ranging from 21 to 61 . The oblique angles were measured on the cross sections of 564 CT scans using engineering software. The angle was measured in such a way that one line was drawn along the inner surface of the lamina in the neuroforamen and the other line bisecting the vertebra and spinous process of the same segment. The angle formed by these 2 lines represents the potential optimal needle entry angle since this needle entrance will likely keep the needle at the posterior wall of the neuroforamen and thus it will less likely hit the vascular or neural structures inside. This angle was taken at each level, i.e., from C3-
4 down to C7-T1 levels. The mean angle obtained was used in guiding subsequent ipsilateral oblique C-arm fluoroscope rotation during cervical transforaminal epidural steroid injections.

Results: In both males and females, the mean entrance angles with a standard deviation (SD) at C2-3, C3-4, C4-5, C56, C6-7, and C7-T1 are as followings: $49 \pm 6,50 \pm 5,50 \pm 4$, $52 \pm 5,53 \pm 5$, and $49 \pm 6$ respectively. Thirty patients with cervical radiculopathy at various levels were injected with the spinal needle entry angle in 50 degree oblique under fluoroscopic guidance. With the patient in a supine position on the procedure table, the head was maintained midline and the shoulders depressed. The C-arm was rotated ipsilaterally into the oblique position at 50 degrees so that the largest cross-sectional area of foramen to be injected was visible. Digital subtraction angiography confirmation was used which confirmed no vascular uptake. Injections were performed successfully without any signs of nerve root or vertebral artery penetration.

Conclusion: It appears that the optimal entry angle for cervical transforaminal epidural injections using the anterior oblique approach lies within the range of 50 degrees. More research studies with larger patient samples must be performed to confirm that the 50 degree oblique angle is the most optimal and safe needle entry angle in cervical transforaminal epidural injections.

Key words: Epidural steroid injection (ESI), transforaminal epidural injections (TEI), cervical transforaminal epidural injections (CTEI), safe entry angle, patient safety and cervical epidural steroid injection, complications to cervical transforaminal epidural steroid injection, steroids 


\title{
14. Detection Rate of Intravascular Injection in Cervical Transforaminal Epidural Steroid Injections using Fluoroscopy with and without Digital Subtraction Angiography.
}

\author{
Boqing Chen MD, PhD, Jeffrey Chacko MD, Fredrick Comrie, MD, and Huey-Jen Lee, MD
}

Center for Advanced Pain Management and Rehabilitation, LLC, Newark, NJ

\begin{abstract}
Objective: To evaluate whether digital subtraction angiography (DSA) combined with real-time fluoroscopic imaging improved the detection rate of intravascular injections during cervical transforaminal epidural steroid injections (CTFESIs) in which real-time fluoroscopic imaging alone did not reveal vascular uptake.
\end{abstract}

Study Design: A retrospective interventional study.

Setting: Outpatient procedure suite in academic private office setting (outpatient surgery center).

Participants: A total of 77 subjects with cervical radicular pain, who had CTFESIs performed between 8/20/09 and $4 / 10 / 10$

Summary of Background Data: CTFESIs are commonly performed to provide symptomatic relief in patients with cervical radiculopathy. These injections carry risks however, including brain and spinal cord infarcts possibly secondary to vascular insult. Studies have shown that after proper needle positioning, observed blood in the needle hub and aspiration can predict intravascular injection during CTFESIs and although specific, they are not sensitive. Live fluoroscopy with contrast injection may enhance the detection of intravascular uptake during CTFESIs as well. A recent study has shown that by adding Digital subtraction angiography (DSA) technology to real-time fluoroscopic imaging, the detection of vascular uptake nearly doubled. That study, however, was performed with 2 separate subject groups (one using real time fluoroscopy with contrast alone and one using fluoroscopy with DSA). In contrast, there have been no studies to date that have evaluated the extent to which DSA in addition to real time fluoroscopy with contrast injection can increase the detection rate of vascular penetration in the same subject group.

Methods: The incidence of intravascular uptake of contrast with fluoroscopy followed by fluoroscopy plus DSA was observed in 77 subjects treated with CTFESIs (144 total CTFESIs at one or 2 cervical levels unilaterally per subject). As has been the practice of one of the authors $(B C)$, images are first reviewed with conventional fluoroscopy and then analyzed under DSA in order to most fully assess for vascular uptake. Each subject had a clinical diagnosis of cervical radiculopathy. For each subject, the injection level was chosen on the basis of the clinical presentation including history, physical examination, EMG/NCS studies, and imaging studies. Using fluoroscopic guidance, a 25 gauge needle was placed into the epidural space using a trans- foraminal approach according to the practice guidelines of the International Spine Intervention Society. Needle tip location was confirmed with anteroposterior and oblique radiographs. Under real-time fluoroscopy, contrast was injected and the needle tip was repositioned appropriately as needed until no vascular uptake was seen with contrast injection. Then immediately following this, contrast was injected using real-time fluoroscopy with DSA and evidence of arterial or venous vascular uptake was documented. Results were subsequently retrospectively reviewed and differentiated between both venous and arterial uptake after review by a board certified neuroradiolgist.

Results: Intravascular injection was detected in 34\% (49/144) of CTFESIs using fluoroscopy alone. The use of fluoroscopy with DSA detected intravascular injection in $51.4 \%$ (74/144) of CTFESIs. Therefore fluoroscopy with DSA increased intravascular detection by $17.4 \%$ (25/144). Furthermore, it was found that intravascular injection was detected in $51.4 \%$ (38/74) of CTFESIs using fluoroscopy plus DSA in which live fluoroscopy without DSA was negative for vascular uptake. This was further divided into venous and arterial uptake. After review by a board certified neuroradiologist, it was found that $100 \%$ (74/74) was venous uptake and 0\% (0/74) was arterial uptake.

Discussion: This is the first study to compare the use of fluoroscopy with contrast alone and fluoroscopy with DSA for the detection of intravascular uptake in the same patient population. The results of this study clearly demonstrated that the use of DSA with fluoroscopy is associated with an increased detection rate of venous intravascular injection during CTFESIs. Although no instances of arterial uptake were found in this study, even with proper needle positioning within the posterior aspect of the cervical neural foramen, there is risk of intravascular penetration which can potentially lead to rare yet serious neurological sequelae, including spinal cord and brain infarction and even death. It has been shown through cadaveric studies that there is great anatomic variation in vascular anatomy around and within the cervical intervertebral foramina. The ascending and deep cervical arteries can contribute radicular or segmental medullary arteries that traverse the posterior aspect of the cervical inter-vertebral foramen and ultimately communicate with the anterior and posterior spinal arteries. These radicular arteries are vulnerable to cannulation by the spinal needle during CTFESIs and inadvertent cannulation of these radicular arteries can lead to severe neurological sequelae. This is thought to be caused by vasospasm or embolization of particulates steroid during intravascular injection. 
Conclusions: Even with proper needle positioning within the cervical neural foramen, there is risk of intravascular penetration. Real-time fluoroscopy with contrast alone can miss intravascular uptake as seen in this study. The use of DSA with realtime fluoroscopy improves the detection rate of intravascular injection during CTFESIs. Although our study failed to reveal arterial uptake, DSA has been shown to detect both arterial and venous intravascular penetration that was missed with fluoros- copy alone. This can potentially prevent catastrophic neurologic sequelae and should reinforce the need for fluoroscopy with real-time DSA during CTFESIs.

Key words: Cervical transforaminal epidural steroid injection; digital subtraction angiography; radicular artery; complication; fluoroscopy 


\title{
15. Conversion of Chronic Pain Patients from Full-Opiate Agonists to Buprenorphine
}

\author{
Jonathan Daitch, MD, Michael Frey, MD, David Silver, and Carol Mitnick, ARNP
}

Advanced Pain Management and Spine Specialists, Fort Myers, FL

Purpose: Suboxone is a sublingual buprenorphine preparation that has been used to treat opiate dependence. Buprenorphine is unique among the opiate-class of analgesics; this compound has a high affinity for the mu-receptor yet only partially activates it. The Drug Enforcement Administration (DEA) has acknowledged the legality of an off-label use to treat pain with the sublingual buprenorphine preparation. Our research goal was to quantify the level of analgesia provided by sublingual buprenorphine, as well as identify patient groups that are most likely to benefit from this therapy.

Methods: Retrospective data from clinical records was taken on 96 chronic pain patients (40 male and 56 female, age 21-81) who had been previously treated with opiate-agonist drugs and were converted to buprenorphine during the study. Data collected from patient profiles included age, sex, diagnosis, medication history, pre-induction opioid intake, reason for detoxification, pre-induction Clinical Opiate Withdrawal Score (COWS), and if applicable, cause of Suboxone discontinuation. Pain levels and Quality of Life (QOL) scores were recorded before and after conversion to buprenorphine.

Results: After initiation of buprenorphine therapy, the mean pain scores on a scale from 0-10, for all patients decreased by 1.4 points. Within the group that continued Suboxone, mean pain scores decreased by 2.4 points $(P<0.001)$. Patient QOL was not significantly affected by buprenorphine therapy. The success rate was highest for patients using fentanyl, hydrocodone, and morphine before Suboxone induction. These patient groups had a $69 \%$ continuation rate for fentanyl and a $60 \%$ continuation rate for hydrocodone and morphine. The highest rate of discontinuation was present in the group of patients who were maintained on oxymorphone before conversion to Suboxone (62\% discontinued). Results indicate that $59 \%$ of patients originally taking between 201-600 mg morphine equivalents per day continued Suboxone therapy, 62\% of patients taking between 101-200 mg morphine equivalents per day continued Suboxone therapy, and $39 \%$ of patients taking between 0-100 mg morphine equivalents per day continued Suboxone therapy. A point biserial correlation test was performed and identifies a $22 \%$ correlation $(P<0.02)$ between pre-induction opioid dose and continuation of buprenorphine therapy.

Conclusion: Patients on higher doses of opiate medication (100-600mg morphine equivalents) seemed to fare better with conversion to Suboxone than patients on lower doses $(<100$ mg morphine equivalents). This may be related to the development of opiate-induced hyperalgesia in the class of high dose opiate users. The group of patients continuing Suboxone therapy for more than 3 months reported significant decreases in pain (2.4 points). The opiate drug used by the patient before Suboxone induction appears to have some effect on Suboxone conversion success. Patients maintained on fentanyl, hydrocodone, or morphine had the highest likelihood of continuation. Despite no significant increase on QOLscale, patients who had a successful conversion to Suboxone indicated a subjective improvement in mental clarity, as well as family relationships. This is a significant trend in this patient population, many of which have become dysfunctional. Further research should investigate which subclasses of chronic pain patients fare better with conversion to Suboxone. 


\section{6. mild@ (Minimally Invasive Lumbar Decompression) Procedure for the Treatment of Lumbar Spinal Stenosis: Procedure Description and Safety}

Timothy R. Deer, MD

Director, The Center for Pain Relief, Clinical Professor, Department of Anesthesiology, West Virginia University, Charleston, WV

Background: Minimally Invasive Lumbar Decompression (mild) is a minimally invasive treatment for pain relief from symptomatic central lumbar canal stenosis. Lumbar spinal stenosis is a common problem that can be caused by many factors, the most common being ligamentum flavum hypertrophy. The mild technique provides removal of small but adequate amounts of ligament and lamina, avoiding the need for aggressive resection of bone and muscle, as well as surgical implants. During the mild procedure, bone and ligament sculpting tools are passed through a small 6G portal, under fluoroscopic visualization, to achieve decompression.

Objective: To describe the mild procedure and to report on safety of this ultra-minimally invasive lumbar decompression method.

Methods: Safety data of the mild procedure has been collected either manually or electronically for over 250 mild patients. All patients were treated with the mild procedure for symptomatic lumbar spinal canal stenosis. mild is conducted through a very small Portal site under fluoroscopic guidance, and is most often performed with moderate sedation. The mild decompression procedure can be performed unilaterally or bilaterally, at single or multiple levels.

Results: Data have been collected and reviewed on over 250 patients treated with the mild procedure. To date, there have been no reports of serious adverse events or major complications related to the mild devices or the procedure in these clinical cases. Major complications are defined as dural tears, nerve root damage, post-op infection, hemodynamic instability, and post-op spinal structural instability. There also have been no reports of blood transfusions, epidural bleeding, or hematomas.

Conclusions: The mild procedure is a safe method for the treatment of lumbar spinal stenosis. No serious device or procedure-related adverse events were reported in over 250 mild patients with follow-up ranging from acute to one year. This minimally invasive procedure allows decompression with minimal surrounding tissue disruption. mild offers an early option for the treatment of lumbar spinal stenosis following failed conservative therapy, but prior to more invasive surgical treatment. 


\title{
17. Buttock Pocket Placement of Implantable Drug Pumps: Overview of a Single Center Experience
}

\author{
David M. Schultz, MD, Margaret Beltrand, RN, Nancy Scholz, RN, Russell Flewellen, BS, \\ CCRC, Anna Von Seggern, BS, Angela M. Greene, MPAS, PA-C, RN
}

Medical Advanced Pain Specialists, Edina, MN

\begin{abstract}
Background: Implantable drug pumps have been invaluable tools in treating intractable pain that does not respond to more conservative treatment. Physicians are instructed to implant these devices in an abdominal pump pocket (APP). APP implants can be impractical for both technical and cosmetic reasons and the pump may rotate or flip within the abdominal panniculus of obese patients. In addition, an APP requires positioning the patient in the lateral position on the operating table which makes AP fluoroscopy, spinal access and catheter anchoring more difficult. Furthermore, lateral positioning dramatically increases operating times when compared to implanting a pump into an upper buttock pump pocket (BPP) site with the patient positioned prone. Historically, with spinal cord stimulation implants primarily utilize an upper buttock pocket site for the internal pulse generator and the surgery is usually performed entirely in the prone position. The newest generation of Medtronic pumps includes the 20cc Synchromed 2 pump which is small enough to fit into a BPP. Accordingly, our practice typically utilizes an upper BPP site. This approach provides both surgical advantages and more stability of the pump within the pocket.
\end{abstract}

Purpose: The purpose of this communication is to describe our experience with BPP implants.

Study Design/Setting: This study is a retrospective medical record review of patients in a single multi-physician practice, Medical Advanced Pain Specialists (MAPS), Minneapolis, MN, who received an implantable drug pump for the 5-year period ending on October 31, 2009.

Patient Sample: Patients receiving a pump between October 2004 and October 2009 were evaluated. Only patients ( $N$ $=252$ ) receiving SynchroMed pumps with a BPP were included in this analysis.

Outcome Measures: Outcome measures included adverse events, - explants and reasons for explants, device years of experience, subjective measures of patient satisfaction.

Methods: The electronic medical record system at MAPS was used to identify a patients meeting the search criteria. All patient clinic visits related to the pump were reviewed for adverse events and explants. Patients were also provided with a questionnaire asking them to provide their subject evaluation of their satisfaction with the pump.

Results: The results of this evaluation are presented in the table.

At $14 \%$, the catheter complication rate is comparable and slightly lower than the $20 \%$ in the published literature. The rate of other adverse events and non-elective explants is also favorable.

\begin{tabular}{|l|l|}
\hline Parameter Measured & Value \\
\hline Demographics & - \\
\hline Number & 252 \\
\hline Male/Female & $108 / 144$ \\
\hline Age - years (St Dev) & $54.1(13.2)$ \\
\hline Follow-Up Time & - \\
\hline Total device years of experience & 483 \\
\hline Mean per patient time with device - years (St Dev) & $1.9(1.4)$ \\
\hline Catheter Complications & - \\
\hline Number of Patients with a Catheter Complication - N (\%) & $35(14 \%)$ \\
\hline Total Number of Catheter Complications & 40 \\
\hline Occlusion/Kinking & $24(9 \%)^{*}$ \\
\hline Dislodgement/Migration & $13(5 \%)^{*}$ \\
\hline Fracture/Break & $1(0.4 \%)$ \\
\hline Cut/Puncture & $1(0.4 \%)$ \\
\hline Disconnected from pump & $1(0.4 \%)$ \\
\hline Other Adverse Events & - \\
\hline Number of Patients with other AEs - N (\%) & $38(15 \%)$ \\
\hline Total Number of AEs & 53 \\
\hline Seroma & $16(4 \%)^{*}$ \\
\hline Infection & $9(4 \%)$ \\
\hline Meningitis & $6(2 \%)$ \\
\hline All Others & $22(8 \%)^{*}$ \\
\hline Explants - N (\%) & $31(12 \%)$ \\
\hline Explants due to AEs & $26(10 \%)$ \\
\hline${ }^{*}$ Multiple episodes of same event occurred in at least one patient (percentage \\
\hline of patients calculated based on number of patients that experienced the event) \\
\hline
\end{tabular}

The procedure was well-tolerated and in general, patient satisfaction with the buttock implant is high. Patients provided positive feedback regarding the cosmetic appearance and utility of this location.

Conclusions: We reviewed records for 252 patients that have received BPP Synchromed pumps in our practice. The data indicate that the procedure is well tolerated and the rate of catheter complications for pumps into an upper BPP site is lower or comparable to the published literature for abdominal pocket site implants. As this was a retrospective analysis, the study was not statistically powered to detect differences with historical controls. A prospective randomized trial would be required to provide definitive evidence of benefit for BPP. However, these data indicate that implantation of pumps in upper BPP is a clinically feasible alternative. 


\title{
18. A Randomized, Placebo Controlled Study to Assess the Efficacy of Lateral Branch Denervation for Chronic Sacroiliac Joint Pain
}

\author{
Andrew Gross, MD \\ Coastal Orthopedics \& Sports Medicine, Bradenton FL
}

Background: The sacroiliac joint (SIJ) has been increasingly implicated as a source of chronic low back pain. It has an injection confirmed prevalence of $13 \%$ to $30 \%$. and has been associated with pain developing post lumbo-sacral fusion. The joint and supporting posterior ligament complex are innervated by lateral branches of the S1-S3 dorsal ramus and the L5 dorsal ramus proper. The location and number of lateral branches is inconsistent making this a challenging structure for radiofrequency denervation. A number of studies have described the use of cooled radiofrequency electrodes to create large volume lesions along the posterior sacrum. Studies have reported success rates (50\% VAS reduction) of 50\% and $64 \%$, respectively, at 3 months follow-up. This study presents preliminary outcomes from a randomized placebo-controlled study on the use of cooled RF to treat sacroiliac joint pain.

Methods: Following ethics review board approval for the study, 51 patients were enrolled and randomized 2:1 (treatment:sham)*. Inclusion criteria included physical examination consistent with $\mathrm{SI}$ joint pain, and $>75 \%$ pain relief from dual lateral branch blocks. Anesthetic Injections were multi-level, multi-depth with max 3cc of lidocane per Dreyfuss et al. Lesions were performed with the SInergy System (Baylis Medical Company, Montreal QC) at S1, S2, S3 and L5 dorsal ramus as described elsewhere. Outcome measures included Visual Analogue Scale (VAS) for pain, SF-36 Bodily Pain (SF-36BP) and SF36 Physical Function (SF-36PF), Oswestry Disability Index (ODI), Global Perceived Effect (GPE), and narcotic consumption. Follow-up data is to be collected at one, 3, 6, 9, and 12 months.

Results: Mean age of patients enrolled was $61 \pm 16$ years.
At the 3-month follow-up, 52\%* of patients in the treatment arm achieved $50 \%$ reduction in VAS pain scores as compared with $29 \%$ * of subjects in the sham group. The mean reduction in VAS in the treatment group was 2.4, as compared with 0.46 in the sham group $(P=0.03)$. The mean SF-36BP increased by 13.71 in treatment group, as compared with a decrease of 5.85 in the sham group $(P=0.02)$. The mean SF-36PF increased by 11.32 in the treatment group, as compared with a decrease of 3.09 in the sham group $(P=0.01)$. Thirty-two percent of patients in the treatment arm experienced a 10 point drop in ODI, $62 \%$ of patients experienced a positive GPE, and $20 \%$ reduced or eliminated their pain medication. In contrast, $0 \%$ of patients in the sham arm experienced a 10 point drop in ODI, 20\% experienced a positive GPE, and 7\% reduced or eliminated their pain medication.

Conclusions: Preliminary data suggest that clinically significant reductions in pain relief are achieved in greater than 50\% of patients treated, with some patients maintaining benefit past 12 months. These results are consistent with previously published results on the use of cooled RF to treat SIJ pain. The results of this study are particularly encouraging for an aging study population.

\footnotetext{
* 3 month data was available for 25 patients in the treatment arm and 14 patients in the sham arm at time of submission
} 


\title{
19. The Influence of Ethnicity on Visual Analog Scale Pain Ratings Pre- and Post- Radiofrequency Application for Pain of Zygapophyseal Joint Origin
}

\author{
Kianfa Martinez-Lu, MD, Schuyler A. Rogg MD, Dan Martin, MD, and William Hammonds, MD
}

Department of Anesthesiology and Perioperative Medicine, Medical College of Georgia, Augusta GA

Objective: Increased scrutiny on the allocation of health care dollars makes the identification of factors influencing treatment outcomes important. Few studies have examined the relationship between ethnicity and the efficacy of chronic pain management interventions. Radiofrequency application (RFA) is a common treatment modality for pain due to zygapophyseal joint (z joint) disease. This survey reviewed the efficacy of RFA in African Americans (AA) and non-Hispanic Caucasians patients.

Design: The present study is a retrospective chart review of patients, who underwent RFA to the the L3, L4 medial branch nerves, dorsal ramus of $L 4$, and the accessory nerve from the $\mathrm{S} 1$ dorsal ramus to the L5-S1 z joint. To qualify for the RFA, all patients had a greater than $50 \%$ reduction in low back pain visual analog scale (VAS) pain scores after neural blockade of the previously mentioned nerves using $0.2 \mathrm{ml}$ of $2 \%$ lidocaine at each level. Patients were followed in the Medical College of Georgia Pain Clinic from 4 to 6 weeks after RFA treatment. A stratified random sample of charts was selected from all AA and Caucasian patients treated during 2009, with 40 Caucasian and 40 AA patients selected for each group. Comparisons between ethnic groups were made using Chi square analysis for the categorical data and 2 sample t-tests for the continuous data. Changes in visual analog scale (VAS) pain scores from pre- to post-RFA were analyzed of covariance (ANCOVA) where changes were adjusted for the baseline VAS prior to making ethnic comparisons. Additional covariates examined include gender, smoking, insurance, psychiatric history, marital status, low back surgery, diabetes type 2 , age, education, body mass index, and opioid consumption. After controlling for baseline
RFA VAS and ethnicity, ANCOVA was used to determine significant differences in the efficacy of RFA treatment caused by the aforementioned covariates.

Results: Both AA and Caucasian patients showed statistically significant improvements in post-RFA VAS scores $(P<0.0001)$ based on paired t-test analysis. Caucasians showed significantly greater benefit with a post-RFA VAS score decrease of $47 \%$ (4.7 units) while AA patients experienced a 13.8\% (1.38 units) post RFA VAS decrease in score (ANCOVA $P<0.0001$ ). Other covariates that were found to be associated with a poorer response to RFA included previous low back surgery $(P=.015)$, and prior psychiatric history $(P=0.039)$. These findings suggest the need to overcome RFA outcome disparities with earlier and more effective complementary treatment strategies for AA.

Conclusions: Based upon these observations, ethnicity appears to influence the effectiveness of RFA treatment for back pain due to $z$ joint disease. In no manner should these results be interpreted as an indication to withhold treatment to patients based on ethnicity. However, the results could suggest that the use of VAS may not be uniformly valid across ethnic groups. These findings underscore the need for continued research on this subject, specifically targeting the measurement of pain ratings and the relationship to functional status. Further research is needed to develop a more comprehensive understanding of these outcome disparities and to develop a crosscultural strategy to improve treatment outcomes. 


\title{
20. Body Injury as Risk Factors for Low Back Radiating Pain in the United States Adult Population
}

\author{
Wenchun $\mathrm{Qu}, \mathrm{MD}, \mathrm{PhD}^{1}$, Mark F. Hurdle, MD¹, Chengwei Wang, MD, MS², and \\ Kevin R. Schmidt, MD²
}

${ }^{2}$ Mayo Clinic, Rochester, Minnesota; ${ }^{2}$ University of Southern California, Los Angeles, CA

Background: Low back pain is a major clinical and public health issue due to its scope and severity. It was reported that $70-85 \%$ of all people have back pain at some time in life. Fifty-one percent of adults in the US experienced low back radiating pain during the past 3 months. As a major burden to the health care system, low back pain was identified by the Institute of Medicine as one of the top 100 topics prioritized for attention and funding. Identification of risk factors is of great importance for recognizing the condition in our clinical encounter as well as implementation of public health preventative measures.

Objective: This research focuses on injuries of body parts as risk factors for low back radiating pain in the US adult population

Design: This is a population-based complex survey study administered annually by the United States Census Bureau for the National Center for Health Statistics, Centers for Disease Control and Prevention.

Methods: Participants were randomly selected based on a multistage stratified sampling design from 2004 to 2008. A sample of participants 18 years of age or older were included in the study. Low back radiating pain was defined as low back pain spreading down either leg or to areas below the knees, which was experienced in the past 3 months, lasting at least one whole day. An injured body part was defined as all medically consulted injury episodes that occurred during the past 3 months.
Results: A total of 1655 participants with injuries severe enough to be medically consulted reported low back radiating pain. Forty-eight percent in those with injuries in the lower extremities reported low back radiating pain, followed by $44 \%$ in those with spine and pelvic injuries, $41 \%$ in chest and abdominal injuries, 39\% in head injuries and 34\% in upper-extremity injuries. After controlling for age and gender, compared with a reference group of respondents with upper extremity injuries, those with lower extremity injuries were 1.6 times $(95 \% \mathrm{Cl} 1.2-$ 2.1) more likely to develop low back radiating pain, while the likelihood was 1.5 times higher $(95 \% \mathrm{Cl} 1.1-1.9)$ in those with spine and pelvic injuries. There is no significant increase in those with injuries in the head (relative risk $1.3 ; 95 \% \mathrm{Cl} 0.7-2.5$ ), nor in the chest or abdomen (relative risk $1.1 ; 95 \% \mathrm{Cl}$ of $0.7-1.7$ ).

Limitations: This study is a cross-section survey research, which obtains injury and low back radiating pain data at the same time point. It would not be appropriate to assume any causal implications. For answering the question of causality, prospective studies might be needed.

Conclusion: Disruption of axial stability might play a major role in the development of low back radiating pain. Injuries in the lower extremity and in the spine or pelvic might indirectly or directly compromise axial stability, thus subjecting individuals to increased risk of low back radiating pain.

Key words: back pain, risk factors, injuries 


\title{
21. Regulation of Calcium Channels and Catecholamine Release by Gabapentin
}

\author{
R. David Todd, MD and Kevin P.M. Currie, PhD \\ Vanderbilt University Medical Center, Department of Anesthesia, Nashville, TN
}

Introduction: Gabapentin is widely prescribed for neuropathic pain states and there is a growing body of literature to support gabapentin's preemptive analgesic effects for post-operative pain. Several animal models have demonstrated that the sympathoadrenal system likely plays a role in the development of hyperalgesia. Interestingly, a recent study showed that pre-treatment with gabapentin was shown to cause blunting of the hemodynamic response to direct laryngoscopy and tracheal intubation. This clinical evidence seems to indicate gabapentin not only modulates neurotransmitter release but might also alter adrenal catecholamine release.

The cellular mechanisms to explain these diverse clinical effects are not clear at this time. Currently, the prevailing hypothesis is that gabapentin exerts its actions through binding to the $\alpha 2 \delta$ subunit of voltage-gated $\mathrm{Ca} 2+$ channels to modulate calcium influx and subsequent neurotransmitter release. Other evidence suggests the effects of gabapentin on neurotransmitter release are more complex. In this study we explored the hypothesis that gabapentin modulates adrenal catecholamine release independently from effects on calcium influx.

Methods: This project is the first to assess the utility of the adrenal chromaffin cell model to investigate the cellular mechanism(s) of gabapentin. In addition to playing a central role in the acute stress response, adrenal chromaffin cells are a widely used neurosecretory model that enables detailed insight into the mechanisms and regulation of neurotransmitter and hormone release. Specifically, we used cellular imaging of calcium entry (FURA-2) and, in parallel experiments, HPLC to assay catecholamine release from dishes of chromaffin cells ("bulk secretion assays"). Elevated $\mathrm{KCl}$ and carbachol (for 5 minutes) was used to depolarize the cells, open voltage-gated calcium channels, and cause intracellular calcium influx. Cells were bathed in various concentrations of gabapentin, $1 \mathrm{mM}$ or $100 \mathrm{uM}$, for either 30 minutes or 24 hours to simulate acute and chronic exposures, respectively. Control cells were tested on the same day and were from the same preparations as experimental cells.
Results: Acute applications of GBP did not alter baseline calcium but produced a small statistically significant increase in peak calcium entry $(1538 \pm 43 \mathrm{nM}, \mathrm{n}=144$, Vs $1682 \pm 45 \mathrm{nM}$, $n=135, P<0.03)$. Furthermore, the decline in intracellular calcium during the 5 -minute $\mathrm{KCl}$ stimulation was reduced by gabapentin from $52 \pm 1 \%$ to $39 \pm 1 \%(P<0.0001)$. Similarly 24 hour treatment with gabapentin increased the peak change in calcium from $1403 \pm 38 \mathrm{nM}$ to $1699 \pm 46 \mathrm{nM}$ and reduced the decline from $44 \pm 1 \%$ to $31 \pm 1 \%(P<0.0001)$ (Fig $1 \mathrm{~A})$. In contrast, 24 hour treatment with gabapentin produced a statistically significant decrease in catecholamine release (Fig 1B). Catecholamine release is also significantly reduced in the presence of gabapentin when a cholinergic stimulant (100 uM carbachol) is used to depolarize cells (data not shown).

Conclusion: Our data shows that gabapentin leads to an increase in intracellular calcium but concomitantly reduced catecholamine release from adrenal chromaffin cells. This differs from the prevailing hypothesis that gabapentin's alteration in calcium influx is the cause for decreased neurotransmitter release but supports our hypothesis that gabapentin likely alters neurotransmitter release independently from its effects on calcium influx at the voltage-gated Ca2+ channel. In addition, our data is the first to support the idea that gabapentin likely exerts some of its physiological and analgesic effects through modulation of sympathoadrenal catecholamine release.

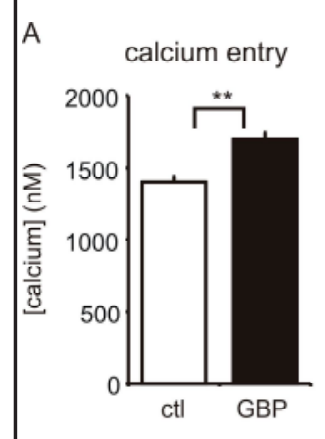

B

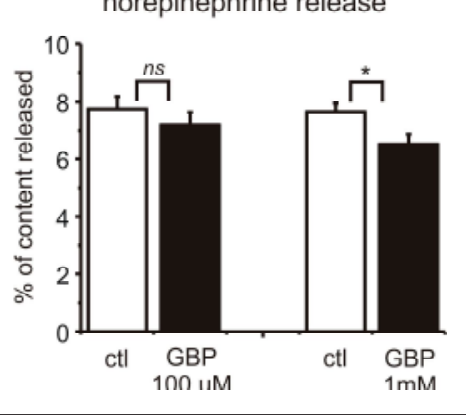




\section{The Use of Intravenous Magnesium for the Treatment of Fibromyalgia \\ LCDR Erik P. Voogd, MD, CAPT Robert J. Mendez, DO, CAPT Michael A. Mazzilli, MD, CDR William A. Beckman, MD, and Thomas J. Moran, DO}

Department of Anesthesiology and Pain Medicine, Naval Medical Center Portsmouth, Portsmouth, VA

Introduction: Fibromyalgia is a painful, often debilitating condition that at this time has no known cure. It is characterized by generalized symptoms including aching pain, morning stiffness, a feeling of swelling in the hands, pain and fatigue upon exercise, and non-restorative sleep.

Multiple treatment modalities attempt to ameliorate these symptoms in an effort to improve the quality of life for patients afflicted with this disorder but long-term relief is rarely achieved. Clinical studies and anecdotal reports have suggested that some patients treated with an oral preparation of magnesium and malic acid (Super Malic $\circledR$, Optimox Corporation, Torrance, CA) may note significant improvement of their symptoms.

Background and Significance: The most common symptoms of fibromyalgia include fatigue, muscle tender points, morning stiffness, and non-restorative sleep. In 1990 the American College of Rheumatology developed several criteria for diagnosing fibromyalgia. These have become the accepted standards for diagnosing and classifying the condition.

Magnesium, a muscle relaxant, is an essential mineral and is involved in multiple enzymatic reactions. Previous research has demonstrated that patients with fibromyalgia are often deficient in intracellular magnesium. This deficiency may be detected in the muscles or red blood cells even though serum levels remain in the normal range. A previous study has shown the possible benefit of oral magnesium and malic acid in reducing patients' pain within 48 hours. The goal of our study was to investigate whether or not intravenous magnesium may lead to relief from the pain of fibromyalgia.

The side effects and risks of intravenous magnesium infusion include drowsiness, depressed reflexes, flaccid paralysis, hypothermia, hypotension, flushing, depressed cardiac function, diaphoresis, and hypocalcemia. Magnesium toxicity may cause heart block, respiratory paralysis, and circulatory collapse. While intravenous magnesium has not been studied for efficacy in treating fibromyalgia, the dosing schedule used in our experimental group is less than the amount used for standard therapy in obstetric patients. They are given a loading dose of 4 grams intravenously over 20 minutes followed by a continuous infusion of 1 to 4 grams per hour. The goal in obstetrics is to get plasma levels into the therapeutic range of $5-7 \mathrm{mg} / \mathrm{dl}$. Minor side effects such as depressed tendon reflexes do not occur until the plasma level is 8 to $10 \mathrm{mg} / \mathrm{dl}$. Serious side effects such as respiratory depression do not occur until plasma levels of 10 to $15 \mathrm{mg} / \mathrm{dl}$ are reached. Cardiac conduction abnormalities and circulatory collapse occur at levels higher than 15 $\mathrm{mg} / \mathrm{dl}$. The dose used in the experimental portion of this protocol (i.e., $600 \mathrm{mg}$ ) is much less than that which causes the side effects described and continuous infusion was not employed. Therefore, the expected incidence of side effects was minimal but monitoring for such occurrences was carried out.

Objective: The objective of this study was to determine whether an intravenous magnesium infusion of $600 \mathrm{mg}$ magnesium sulfate would be effective in temporarily relieving the painful symptoms of chronic fibromyalgia. Specifically, our hypothesis was that intravenous magnesium would be more effective in treating the pain of fibromyalgia than normal saline. We further proposed that treatment with intravenous magnesium may offer more rapid and potentially longer lasting relief than oral preparations.

Methods: A prospective, randomized, double-blind, placebo-controlled study was performed in our clinic. All enrollees had been referred to our clinic by a rheumatologist and had a formal diagnosis of fibromyalgia per the American College of Rheumatology criteria for fibromyalgia syndrome. Patients in the treatment arm received $600 \mathrm{mg}$ magnesium sulfate in 50 $\mathrm{mL}$ of normal saline infused intravenously over 20 - 30 minutes on 2 occasions 2 weeks apart. Pain scores were assessed using a $10 \mathrm{~cm}$ visual analog scale (VAS) with 0 at one end (no pain) and 10 at the other end (worst possible pain). These scores were recorded immediately before each infusion and at 4 and 6 weeks after the first infusion for a total of 4 measurements over 6 weeks.

Results: Of the 70 subjects that were randomized and originally enrolled in the study, 63 elected to participate and received a single infusion of drug or normal saline (week 0). Fifty-three subjects received a second dose (week 2). Thirty-eight subjects (18 drug / 20 placebo) elected to submit their VAS scores at week 4 . And lastly, 30 subjects (13 drug / 17 placebo) submitted their VAS scores at week 6 . No adverse reactions were noted in either arm of the study.

We computed a repeated measures Analysis of Variance (ANOVA) on the VAS scores at 0, 2, 4, and 8 weeks comparing the magnesium vs. saline groups (Fig. 1). There was no difference between the 2 groups $(2=.789)$. There was no change over time $(P=.786)$ and no interaction effect $(P=.642)$. The data are shown in Table 1. In order to increase the sample size we computed a repeated measures ANOVA on just the first 4 weeks (Fig. 2). There was no difference between the 2 groups $(P=.859)$, no change over time $(P=.794)$, and no interaction effect $(P=.853)$. The data are shown in Table 2 . 
Table 1.

\begin{tabular}{|c|c|c|c|c|c||}
\hline Group & Time & Mean & Std. Error & \multicolumn{2}{|c|}{ 95\% Confidence Interval } \\
\hline & & & & Lower Bound & Upper Bound \\
\hline Magnesium & 0 & 5.68 & 0.62 & 4.40 & 6.95 \\
\hline & 2 & 5.34 & 0.55 & 4.21 & 6.47 \\
\hline & 4 & 5.24 & 0.69 & 3.83 & 6.64 \\
\hline & 8 & 5.60 & 0.78 & 4.00 & 7.20 \\
\hline Normal Saline & 0 & 5.59 & 0.54 & 4.48 & 6.71 \\
\hline & 2 & 5.52 & 0.48 & 4.54 & 6.51 \\
\hline & 4 & 5.45 & 0.60 & 4.23 & 6.68 \\
\hline & 8 & 6.04 & 0.68 & 4.63 & 7.44 \\
\hline
\end{tabular}

Table 2.

\begin{tabular}{|c|c|c|c|c|c|}
\hline Group & Time & Mean & Std. Error & \multicolumn{2}{|c|}{ 95\% Confidence Interval } \\
\hline & & & & $\begin{array}{c}\text { Lower } \\
\text { Bound }\end{array}$ & $\begin{array}{c}\text { Upper } \\
\text { Bound }\end{array}$ \\
\hline Magnesium & 0 & 5.88 & 0.49 & 4.89 & 6.88 \\
\hline & 2 & 5.65 & 0.45 & 4.75 & 6.55 \\
\hline & 4 & 5.71 & 0.55 & 4.58 & 6.83 \\
\hline Normal Saline & 0 & 5.73 & 0.46 & 4.79 & 6.67 \\
\hline & 2 & 5.51 & 0.42 & 4.65 & 6.37 \\
\hline & 4 & 5.70 & 0.53 & 4.64 & 6.76 \\
\hline
\end{tabular}

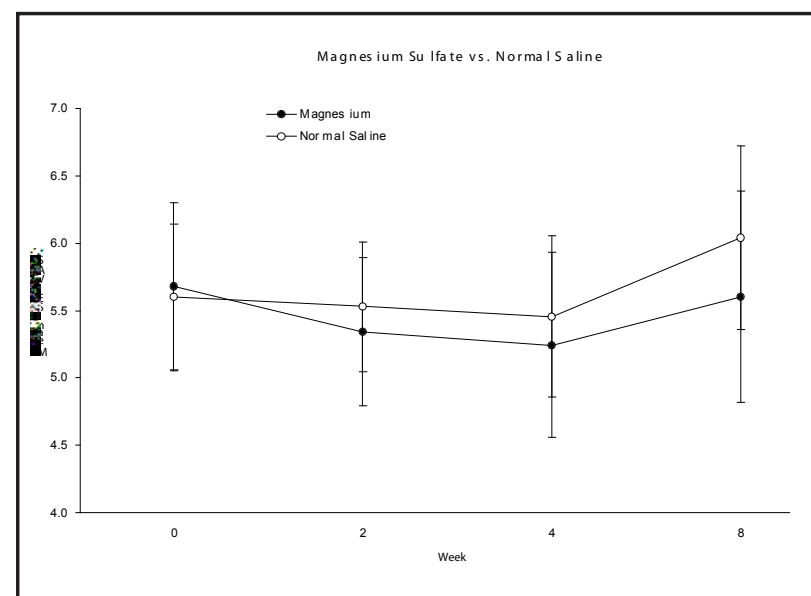

Figure 1.
Conclusion: An intravenous administration of 600 mg magnesium sulfate does not produce a statistically significant improvement in self-reported VAS scores of randomly selected and assigned fibromyalgia patients in a randomized, placebo-controlled, double-blinded trial.

While previous publications (a case series and a single RCT by Ali et al) suggested that patients improved with intravenous infusion of the so-called "Myers' Cocktail" (IVMT), the former only gave an anecdotal example of a single case of fibromyalgia treated with the IVMT and the latter failed to demonstrate a statistically significant difference in VAS scores between the treatment and placebo groups. In the Ali study they used 1 gram of magnesium chloride in their IVMT while we utilized 600 mg of magnesium Sulfate. Therefore, their dose of magnesium was almost twice the amount that we used (1 $\mathrm{g}$ magnesium sulfate $=0.8 \mathrm{~g}$ magnesium chloride). Despite this significantly larger dose of magnesium, as well as the addition of multiple other nutrients, they failed to clearly demonstrate patient VAS score improvement from the cocktail infusion. The authors did, however, express concern that their sample size may have been inadequate to find a significant difference between their treatment and placebo groups since they had noted a powerful placebo effect in their data. In our similar sized study, we noted no significant placebo effect and still were not able to find a statistically significant difference between our treatment and placebo groups.

While the authors can make no statements as to the efficacy of IVMT in the treatment of fibromyalgia syndrome we believe that we have adequately demonstrated that the intravenous infusion of magnesium sulfate alone in the dose range we tested is not in itself a useful treatment for fibromyalgia.

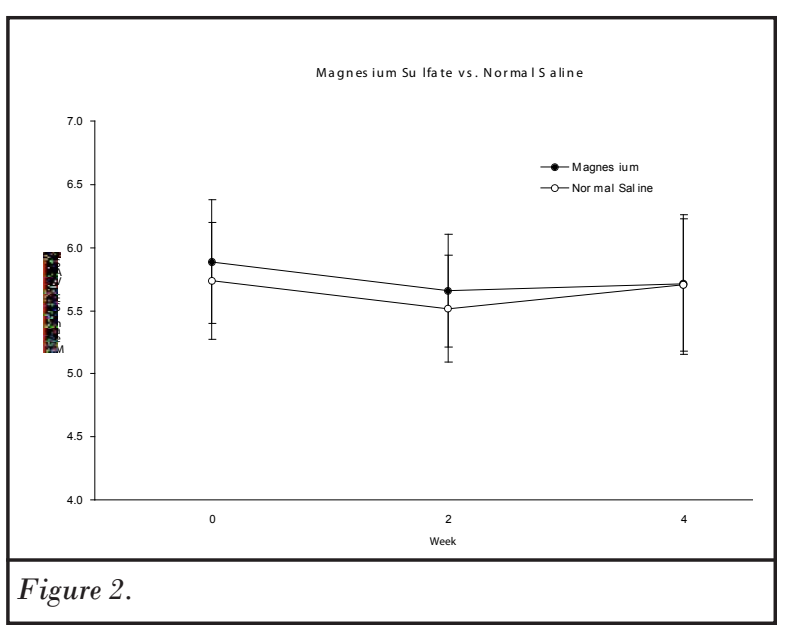

